\title{
Yet More Elements in the Shafarevich-Tate Group of the Jacobian of a Fermat Curve
}

\author{
Benjamin Levitt and William McCallum
}

\begin{abstract}
For certain irregular primes $p$ we construct non-trivial elements in the Shafarevich-Tate group of the jacobian of a quotient of the Fermat curve $x^{p}+y^{p}=1$. These elements are different in general from elements previously constructed by McCallum and McCallum-Tzermias.
\end{abstract}

\section{Introduction}

For an odd prime number $p$ and an integer $s$ with $1 \leq s \leq p-2$, the complete nonsingular curve $F_{s}$ with equation

$$
y^{p}=x^{s}(1-x)
$$

is a quotient of the Fermat curve $x^{p}+y^{p}=1$. It has genus $(p-1) / 2$ and its jacobian $J$ has complex multiplication by $\mathbb{Z}\left[\mu_{p}\right]$, where $\mu_{p}$ is the group of $p$-th roots of unity in $\overline{\mathbb{Q}}$. In this paper we construct non-trivial elements in the $p$-torsion of the Shafarevich-Tate group

$$
\amalg=\amalg\left(J, \mathbb{Q}\left(\mu_{p}\right)\right)
$$

for certain irregular primes $p$. These are new elements, in the sense that there is not in general any linear dependence relation over $\mathbb{Z}\left[\mu_{p}\right]$ between them and ones previously constructed [McC88], [MT03]. This result is of interest because a Selmer group calculation shows that, if $\mathfrak{p}$ is the prime ideal in $\mathbb{Z}\left[\mu_{p}\right]$ above $p$, then

$$
\operatorname{rank}_{\mathbb{Z} / p \mathbb{Z}} \amalg / \mathfrak{p}+\operatorname{rank}_{\mathbb{Z} / p \mathbb{Z}} J\left(\mathbb{Q}\left(\mu_{p}\right)\right) / \mathfrak{p}
$$

is relatively large: heuristically, it is asymptotic to $p / 4$ as $p$ grows [McC92, Theorem 1 and introductory discussion]. Thus we should be able to find either an abundance of $\mathbb{Z}\left[\mu_{p}\right]$-independent elements in $\amalg$ or an abundance of $\mathbb{Z}\left[\mu_{p}\right]$-independent points in $J\left(\mathbb{Q}\left(\mu_{p}\right)\right)$. The current result adds to the growing body of circumstantial evidence that it easier to do the former. Indeed, modulo torsion, the only systematically occurring explicitly known infinite $\mathbb{Z}\left[\mu_{p}\right]$-submodule of $J\left(\mathbb{Q}\left(\mu_{p}\right)\right)$ is the one generated by the Gross-Rohrlich point [GR78].

Consider the usual descent sequence

$$
0 \rightarrow J\left(\mathbb{Q}\left(\mu_{p}\right)\right) / \lambda^{k} J\left(\mathbb{Q}\left(\mu_{p}\right)\right) \rightarrow S_{\lambda^{k}} \rightarrow \amalg\left[\lambda^{k}\right] \rightarrow 0,
$$

2000 Mathematics Subject Classification. Primary 11G30; Secondary 14G25, 14 K15.

Key words and phrases. Shafarevich-Tate group, Fermat curve, Jacobian.

The authors were supported in part by NSF grant DUE 0525009. 
FIGURE 1. Reduction types of $F_{s}$

Tame

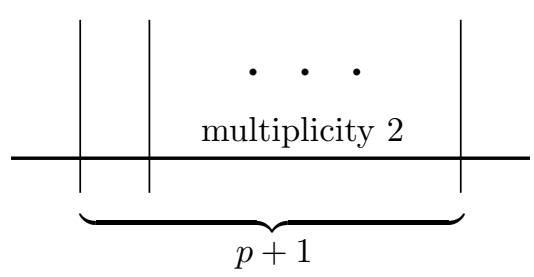

Wild

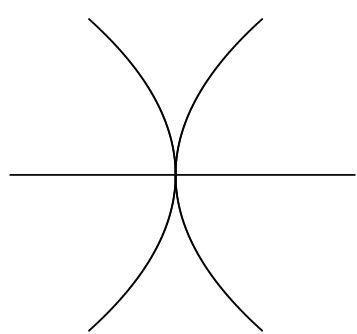

where $\lambda$ is a generator of $\mathfrak{p}$ and $S_{\lambda^{k}}=S_{\lambda^{k}}\left(J, \mathbb{Q}\left(\mu_{p}\right)\right)$ is the Selmer group associated with a positive integer power $\lambda^{k}$. A choice of group isomorphism between $J[\lambda]$ and the group $\mu_{p}$ of $p$-th roots of unity enables us to identify $S_{\lambda}\left(J, \mathbb{Q}\left(\mu_{p}\right)\right)$ with a subgroup of $\mathbb{Q}\left(\mu_{p}\right)^{\times} / \mathbb{Q}\left(\mu_{p}\right)^{\times p}$. The two previous non-triviality results and the one we prove in this paper depend on finding a specific element $\eta \in \mathbb{Q}\left(\mu_{p}\right)^{\times} / \mathbb{Q}\left(\mu_{p}\right)^{\times p}$ which is contained in $S_{\lambda}$ under this identification and, for some $k$, lifts to an element of $S_{\lambda^{k}}$ whose image in $\amalg$ is non-trivial.

The elements $\eta$ come from cyclotomic units, as follows. Let $\Delta=\operatorname{Gal}\left(\mathbb{Q}\left(\mu_{p}\right) / \mathbb{Q}\right)$, let $\omega: \Delta \rightarrow \mathbb{Z}_{p}^{\times}$be the Teichmüller character, and define for any integer $i$ the usual idempotent

$$
\epsilon_{i}=\frac{1}{p-1} \sum_{\sigma \in \Delta} \omega^{-i}(\sigma) \sigma .
$$

Fix a primitive $p$-th root of unity $\zeta$, and define $\eta_{i} \in \mathbb{Q}\left(\mu_{p}\right)^{\times} / \mathbb{Q}\left(\mu_{p}\right)^{\times p}$ by ${ }^{1}$

$$
\eta_{i}=(1-\zeta)^{\epsilon_{i}} \text {. }
$$

If $i$ is even, $2 \leq i \leq p-3$, and $p$ does not divide the Bernoulli number $B_{i}$, then $\eta_{i}$ is locally non-trivial at $p$ (that is, its image in $\mathbb{Q}_{p}\left(\mu_{p}\right)^{\times} / \mathbb{Q}_{p}\left(\mu_{p}\right)^{\times p}$ is nonzero) [Was97, Chapter 8]. Furthermore, by eigenvalue considerations the nontrivial elements among the $\eta_{i}$ with $2 \leq i \leq p-1$ are linearly independent.

We first recall the nontriviality results of McCallum and McCallum-Tzermias. These depend on computing the Cassels pairing of certain elements. The Cassels pairing

$$
\amalg \times \amalg \rightarrow \mathbb{Q} / \mathbb{Z} .
$$

is skew-symmetric and its kernel is the infinitely divisible subgroup of $\amalg$ (a subgroup which is conjectured to be trivial). Its definition is reviewed in [McC88]. The computation depends on local $p$-adic analytic approximations of functions on $F_{s}$, and these approximations depend on a minimal regular model for $F_{s}$ over $\mathbb{Z}_{p}\left[\mu_{p}\right]$. The special fiber for such a model is a curve over the finite field $\mathbb{F}_{p}$ with $p$ elements, and has two possible geometric types, wild and tame, shown in Figure 1. The terminology corresponds to the ramification type of a field of good reduction for $F_{s}$. The wild type is further divided into split and non-split, according to whether the two

\footnotetext{
${ }^{1}$ Note that the notation is different from that used in [MS03]. The element $\eta_{i}$ defined there is equal to the element $\eta_{p-i}$ defined here.
} 
tangent components are defined over $\mathbb{F}_{p}$ or conjugate over a quadratic extension. The reduction type can be computed as follows. For a rational number $x$ relatively prime to $p$ let $q(x)=\left(x^{p-1}-1\right) / p$, and let $\epsilon$ be the Legendre symbol

$$
\epsilon=\left(\frac{2 s(s+1) q\left(s^{s} /(s+1)^{s+1}\right)}{p}\right) .
$$

Then the reduction type of $F_{s}$ is

$$
\begin{array}{ll}
\text { tame } & \text { if } \epsilon=0 \\
\text { wild split } & \text { if } \epsilon=1 \\
\text { wild non-split } & \text { if } \epsilon=-1 .
\end{array}
$$

The first nontriviality theorem that we want to recall here is

THEOREM 1.1 (McCallum [MT03]). Suppose $p$ and $F_{s}$ satisfy the following conditions:

(1) $p \equiv 1(\bmod 4)$

(2) $p \nmid B_{(p-1) / 2} B_{(p+3) / 2}$

(3) $F_{s}$ has wild split reduction at $\mathfrak{p}$.

Then the image in $\amalg$ of the subgroup of $S_{\lambda}$ generated by $\eta_{(p-1) / 2}$ and $\eta_{(p+3) / 2}$ is isomorphic to $(\mathbb{Z} / p \mathbb{Z})^{2}$.

An example of a curve satisfying the conditions of Theorem 1.1 is $y^{17}=x(1-x)$. The proof of the theorem is a calculation of the Cassels pairing between the images of $\eta_{(p-1) / 2}$ and $\eta_{(p+3) / 2}$ in $\amalg[\lambda]$.

The next theorem rests on an extension of the methods in the previous theorem to a calculation of the Cassels pairing between $\amalg[\lambda]$ and $\amalg\left[\lambda^{3}\right]$.

TheOREM 1.2 (McCallum-Tzermias [MT03]). Suppose that $p$ and s satisfy the following conditions:

(1) $p \geq 19$ is regular and $p \equiv 3(\bmod 4)$

(2) $F_{s}$ has tame or wild non-split reduction at $\mathfrak{p}$

(3) s satisfies the congruence

$$
\begin{aligned}
& \left.\qquad\left(s^{s} /(s+1)^{s+1}\right)\right)^{3}-s(s+1) B_{p-3} \not \equiv 0 \quad(\bmod p), \\
& \text { where } q(x)=\left(x^{p-1}-1\right) / p .
\end{aligned}
$$

Then $\eta_{(p+5) / 2}$ lifts to an element of $S_{\lambda^{3}}$, and this element and the element $\eta_{(p+1) / 2} \in$ $S_{\lambda}$ have $\mathbb{Z}$-independent nontrivial images in $\amalg$.

An example of a curve satisfying the conditions of Theorem 1.2 is

$$
y^{19}=x^{2}(1-x) .
$$

In this paper we prove a new non-triviality result. Since $J$ has good reduction outside $p$ and $\operatorname{deg} \lambda=p, S_{\lambda^{k}}$ is contained in $H^{1}\left(G, J\left[\lambda^{k}\right]\right)$, where $G$ is the Galois group of the maximal extension of $\mathbb{Q}\left(\mu_{p}\right)$ unramified outside $p$. Our result makes use of the cup product pairing

$$
H^{1}\left(G, \mu_{p}\right) \times H^{1}\left(G, \mu_{p}\right) \rightarrow H^{2}\left(G, \mu_{p}\right) \otimes \mu_{p},
$$

which gives rise to a pairing

$$
\langle,\rangle: E / E^{p} \times E / E^{p} \rightarrow H^{2}\left(G, \mu_{p}\right) \otimes \mu_{p}=C / p C \otimes \mu_{p}
$$


where $E$ is the group of $p$-units and $C$ is the ideal class group of $\mathbb{Z}\left[\mu_{p}\right]$. This pairing was studied in [MS03] and shown to be nontrivial for $p=37$. Sharifi subsequently showed the non-triviality of the pairing for $p \leq 1,000$ [Sha07].

THEOREM 1.3. Suppose that $p, s$, and $r$ satisfy the following conditions

(1) $r$ is even and $2 \leq r \leq(p+1) / 2$

(2) $F_{s}$ has wild non-split or tame reduction at $\mathfrak{p}$

(3) $\left\langle\eta_{p-r+3}, \eta_{p-3}\right\rangle \neq 0$ (which implies $p \mid B_{r}$ ).

Then $\eta_{p-r+3}$ lifts to an element of $S_{\lambda^{3}}$ whose image in $\amalg$ is nontrivial.

Note that condition (3) implies that $p$ must be an irregular prime for Theorem 1.3 to apply. An example of a curve satisfying the conditions of Theorem 1.3 is $y^{691}=x(1-x)$. It has wild non-split reduction, and 691 divides both $B_{12}$ and $B_{200}$. Both $r=12$ and $r=200$ satisfy condition (2), and $\left\langle\eta_{682}, \eta_{688}\right\rangle$ and $\left\langle\eta_{494}, \eta_{688}\right\rangle$ are both non-zero [MS03, Sha07]. This gives us two independent elements of order 691 in $\amalg$.

We would like to thank the referee for several useful comments and corrections.

\section{Galois structure of the $\lambda^{4}$-torsion}

The divisor $(0,0)-\infty$ on $F_{s}$ is fixed by $\zeta$ and therefore represents a nontrivial $\mathbb{Q}$-rational $\lambda$-torsion point. Let $K=\mathbb{Q}\left(\mu_{p}\right)$. Greenberg determined the field of definition of the higher $\lambda$-torsion:

Theorem 2.1 (Greenberg [Gre81]). We have

$$
K\left(J\left[\lambda^{3}\right]\right)=K
$$

and

$$
K\left(J\left[\lambda^{4}\right]\right)=K\left(\eta_{p-3}^{1 / p}\right)
$$

The first part is Theorem 1 of the cited reference. Although the second part is not explicitly stated, the proof is contained in the first paragraph of Section 5 . (The condition $\omega^{i}(a+1)=\omega^{i}(a)+1$ stated in the reference boils down to $(s+1)^{3} \equiv s^{3}+1$ $(\bmod p)$, which is trivially satisfied.)

Theorem 2.1 enables us to determine explicitly the structure of $J\left[\lambda^{4}\right]$ as a Galois module over $K$. Following [MT03], for $i=1,2,3,4$, we choose a point $P_{i}$ of exact order $\lambda^{i}$ on $J$ so that $\lambda P_{i}=P_{i-1}$ for $i=2,3,4$. These points form a basis for $J\left[\lambda^{4}\right]$ as a vector space over $\mathbb{Z} / p \mathbb{Z}$. Furthermore, $P_{3}$ is a $\lambda^{3}$-torsion point, and therefore defined over $K$, and $\lambda$ itself is defined over $K$. Therefore, for $\sigma \in \operatorname{Gal}(\overline{\mathbb{Q}} / K)$

$$
\sigma\left(P_{4}\right)=P_{4}-\chi(\sigma) P_{1}
$$

for some isomorphism $\chi: \operatorname{Gal}\left(K\left(\eta_{p-3}^{1 / p}\right) / K\right) \rightarrow \mathbb{Z} / p \mathbb{Z}$.

LEMma 2.2. Let $\chi$ be defined by (2.1). We have a commutative diagram

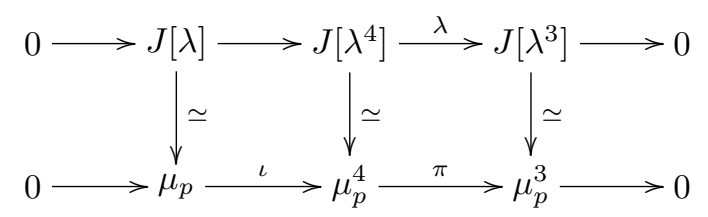


in which $\iota$ is the embedding via the fourth coordinate and $\pi$ is projection onto the first three coordinates. Furthermore, if we let $\sigma \in \mathrm{Gal}(\overline{\mathbb{Q}} / K)$ act on $\mu_{p}^{4}$ by

$$
\left(\begin{array}{cccc}
1 & 0 & 0 & 0 \\
0 & 1 & 0 & 0 \\
0 & 0 & 1 & 0 \\
\chi(\sigma) & 0 & 0 & 1
\end{array}\right)
$$

and on all other modules in the natural way, then the diagram commutes with the action of $\operatorname{Gal}(\overline{\mathbb{Q}} / K)$.

Proof. For $i \leq 4$, let $e_{\lambda^{i}}(P, Q)$ be the $\lambda^{i}$ Weil pairing on $J\left[\lambda^{i}\right]$, as defined, for example, in [McC88]. For any two commuting isogenies $\phi$ and $\psi$ of $J$, the Weil pairing satisfies

$$
e_{\phi}(\psi P, Q)=e_{\phi}(P, \hat{\psi} Q),
$$

where $\hat{\psi}$ is the dual isogeny[McC88, (1.7) and (1.8)]. Define an isomorphism $J\left[\lambda^{i}\right] \simeq \mu_{p}^{i}$ by

$$
Q \mapsto\left(e_{\lambda^{i}}\left(Q, P_{1}\right), \ldots, e_{\lambda^{i}}\left(Q, P_{i}\right)\right) .
$$

Our choice of $P_{i}$ and $\chi$ mean that

$$
\sigma Q=Q-\chi(\sigma) \lambda^{3} Q, \quad Q \in J\left[\lambda^{4}\right]
$$

Therefore, if $1 \leq j \leq i \leq 4$,

$$
\begin{aligned}
e_{\lambda^{i}}\left(\sigma Q, P_{j}\right) & =e_{\lambda^{i}}\left(Q, P_{j}\right)-\chi(\sigma) e_{\lambda^{i}}\left(\lambda^{3} Q, P_{j}\right) \\
& =e_{\lambda^{i}}\left(Q, P_{j}\right)-\chi(\sigma) e_{\lambda^{i}}\left(Q, \hat{\lambda}^{3} P_{j}\right) \\
& =e_{\lambda^{i}}\left(Q, P_{j}\right)+\chi(\sigma) e_{\lambda^{i}}\left(Q, \lambda^{3} P_{j}\right) \\
& = \begin{cases}e_{\lambda^{i}}\left(Q, P_{j}\right)+\chi(\sigma) e_{\lambda^{i}}\left(Q, P_{1}\right) & j=4 \\
e_{\lambda^{i}}\left(Q, P_{j}\right) & j<4 .\end{cases}
\end{aligned}
$$

Here we have used the facts that $\hat{\lambda}=\bar{\lambda}$, the complex conjugate of $\lambda$, and that $\bar{\lambda}^{3} \equiv-\lambda^{3}\left(\bmod \lambda^{4}\right)$.

\section{Selmer Groups}

The Selmer group $S_{\lambda^{i}}$ is defined by exactness of

$$
0 \rightarrow S_{\lambda^{i}} \rightarrow H^{1}\left(K, J\left[\lambda^{i}\right]\right) \rightarrow \sum_{v} H^{1}\left(K_{v}, J\right)
$$

where the sum is over a complete set of valuations of $K$. We summarize here the basic facts about these Selmer groups, and refer the reader to [McC88] and [MT03] for details.

The isomorphism

$$
J[\lambda] \simeq \mu_{p}
$$

chosen in Lemma 2.2 identifies $S_{\lambda}$ with a subgroup of $K^{\times} / K^{\times p}$ defined by local conditions at each valuation of $K$. For every valuation except the unique $p$-adic one, the local condition on $x \in K^{\times} / K^{\times p}$ is simply that it be a local unit modulo $p$-th powers. For the valuation corresponding to the unique prime $\mathfrak{p}$ of $K$ above $p$, Faddeev calculated the local condition, and found that in the wild non-split and tame cases it is

$$
x_{\mathfrak{p}} \in 1+\mathfrak{p}^{(p+3) / 2} \mathcal{O}_{\mathfrak{p}} \quad\left(\bmod K_{\mathfrak{p}}^{\times p}\right)
$$


(See [Fad61] or [McC88] for the calculation.) Let $\eta_{i} \in K^{\times} / K^{\times p}$ be the element defined by (1.1). By construction it is an eigenvector for the action of $\operatorname{Gal}(K / \mathbb{Q})$ with character $\omega^{i}$. On the other hand, for $k \geq 1$, the action of $\operatorname{Gal}(K / \mathbb{Q})$ on $\left(1+\mathfrak{p}^{k} \mathcal{O}_{\mathfrak{p}}\right) /\left(1+\mathfrak{p}^{k+1} \mathcal{O}_{\mathfrak{p}}\right)$ is through the character $\omega^{k}$. Thus

$$
\eta_{i} \in 1+\mathfrak{p}^{i} \mathcal{O}_{\mathfrak{p}} \quad\left(\bmod K_{\mathfrak{p}}^{\times p}\right), \quad 2 \leq i \leq p-3 .
$$

It follows from (3.1) that $\eta_{i} \in S_{\lambda}$ if $(p+3) / 2 \leq i \leq p-3$. We next show that these elements lift to $S_{\lambda^{3}}$. As before, the local condition at every valuation other than the $p$-adic one is that an element be a unit mod $p$-th powers in each component. The local condition at $\mathfrak{p}$ is harder to determine.

For $i=1,2,3$, the Galois isomorphisms $J\left[\lambda^{i}\right] \simeq \mu_{p}^{i}$ chosen in Lemma 2.2 identify $H^{1}\left(K, J\left[\lambda^{i}\right]\right)$ with a subgroup of $\left(K^{\times} / K^{\times p}\right)^{i}$. It is shown in [MT03, Section 2] that the local descent maps

$$
d_{k}: J\left(K_{\mathfrak{p}}\right) / p J\left(K_{\mathfrak{p}}\right) \rightarrow H^{1}\left(K_{\mathfrak{p}}, J\left[\lambda^{i}\right]\right)=\left(K_{\mathfrak{p}}^{\times} / K_{\mathfrak{p}}^{\times p}\right)^{i}
$$

can be written as

$$
d_{k}=\prod_{j=1}^{k} \iota_{P_{j}}
$$

where the maps

$$
\iota_{P_{j}}: J\left(K_{\mathfrak{p}}\right) / p J\left(K_{\mathfrak{p}}\right) \rightarrow K_{\mathfrak{p}}^{\times} / K_{\mathfrak{p}}^{\times p}
$$

are defined by evaluating certain functions $f_{j}$ on the curve at divisors representing points on the Jacobian. (The divisor of the function $f_{j}$ is $p$ times a divisor representing the point $P_{j}$.) We have

$$
\iota_{P_{j}}(\lambda x)=\iota_{P_{j-1}}(x) \text { for } j=2,3 .
$$

LEMma 3.1. If $C$ has wild non-split or tame reduction and $\frac{p+5}{2} \leq i \leq p-3$ then the element

is contained in the Selmer group $S_{\lambda^{3}}$.

$$
\left(\eta_{i}, 1,1\right) \in\left(K^{\times} / K^{\times p}\right)^{3}
$$

Proof. Since $\eta_{i}$ defines a cocycle which is unramified outside the primes above $p,\left(\eta_{i}, 1,1\right)$ satisfies all the local conditions for membership in $S_{\lambda^{3}}$ except the one at $\mathfrak{p}$. Thus membership in $S_{\lambda^{3}}$ is equivalent to being in the image of the local descent map at $\mathfrak{p}$

$$
d_{3}: J\left(K_{\mathfrak{p}}\right) \rightarrow H^{1}\left(K_{\mathfrak{p}}, J\left[\lambda^{3}\right]\right)=\left(K_{\mathfrak{p}}^{\times} / K_{\mathfrak{p}}^{\times p}\right)^{3} .
$$

By [MT03, Proposition 4.1] we can choose $a \in J\left(K_{\mathfrak{p}}\right) / p J\left(K_{\mathfrak{p}}\right)$ such that $\iota_{P_{1}}(a)=$ $\eta_{i}$, and by applying the necessary idempotent we can suppose that $a$ is an eigenvector for the action of $\operatorname{Gal}(K / \mathbb{Q})$, in which case it is in the $\omega^{i}$ eigenspace by $[$ MT03, $(4.2)]$

Consider $d_{3}(a)=\left(\eta_{i}, \iota_{P_{2}}(a), \iota_{P_{3}}(a)\right)$. To prove the lemma, we will find $b \in$ $J\left(K_{\mathfrak{p}}\right) / p J\left(K_{\mathfrak{p}}\right)$ such that $d_{2}(b)=\left(\iota_{P_{2}}(a)^{-1}, \iota_{P_{3}}(a)^{-1}\right)$. Then, by (3.3),

$$
d_{3}(a+\lambda b)=d_{3}(a) \cdot\left(1, d_{2}(b)\right)=\left(\eta_{i}, 1,1\right),
$$

showing that $\left(\eta_{i}, 1,1\right)$ satisfies the local condition at $\mathfrak{p}$. Now, Proposition 4.1 and equation (4.2) of [MT03] show that if $i \geq(p+5) / 2$ then

$$
\iota_{P_{2}}(a), \iota_{P_{3}}(a) \in 1+\mathfrak{p}^{(p+1) / 2} \mathcal{O}_{\mathfrak{p}} .
$$

It follows from [MT03, Proposition 4.2] that we can choose $b \in J\left(K_{\mathfrak{p}}\right)$ so that $d_{2}(b)=\left(\iota_{P_{2}}(a)^{-1}, \iota_{P_{3}}(a)^{-1}\right)$, as we wanted. 


\section{Proof of Theorem 1.3}

Suppose now that we are given $p, r$, and $s$ satisfying the conditions of Theorem 1.3. Conditions (1) and (2) imply $\left(\eta_{p-r+3}, 1,1\right) \in S_{\lambda^{3}}$, by Lemma 3.1.

Since $J$ has good reduction outside $\mathfrak{p}$ and $\operatorname{deg} \lambda=p$, we can regard the Selmer groups $S_{\lambda^{i}}$ as subgroups of $H^{1}\left(G, J\left[\lambda^{i}\right]\right)$, where $G$ is the Galois group of the maximal extension of $K$ unramified outside $\mathfrak{p}$. Regarding $\left(\eta_{p-r+3}, 1,1\right)$ as an element of $H^{1}\left(G, J\left[\lambda^{3}\right]\right)$, we claim that its coboundary in the $G$-cohomology of

$$
0 \rightarrow J[\lambda] \rightarrow J\left[\lambda^{4}\right] \rightarrow J\left[\lambda^{3}\right] \rightarrow 0
$$

is equal to a non-zero multiple of $\left\langle\eta_{p-r+3}, \eta_{p-3}\right\rangle$ under the identification $H^{2}(G, J[\lambda]) \simeq$ $H^{2}\left(G, \mu_{p}\right)$. This can be seen as follows.

In this paragraph we use additive notation for the abelian group $\mu_{p}$, and we use the diagram and notation of Lemma 2.2. Since $\mu_{p}^{3}$ is fixed by $G$, our element $\left(\eta_{p-r+3}, 1,1\right) \in H^{1}\left(G, \mu_{p}^{3}\right)$ is represented by a homomorphism $(x, 0,0): G \rightarrow \mu_{p}^{3}$, which we lift to the cochain

$$
\bar{x}=(x, 0,0,0): G \rightarrow \mu_{p}^{4} .
$$

Then

$$
\begin{aligned}
\delta(\bar{x})(\sigma, \tau) & =\left(\begin{array}{c}
x(\sigma \tau) \\
0 \\
0 \\
0
\end{array}\right)-\left(\begin{array}{c}
x(\sigma) \\
0 \\
0 \\
0
\end{array}\right)-\left(\begin{array}{cccc}
1 & 0 & 0 & 0 \\
0 & 1 & 0 & 0 \\
0 & 0 & 1 & 0 \\
\chi(\sigma) & 0 & 0 & 1
\end{array}\right)\left(\begin{array}{c}
x(\tau) \\
0 \\
0 \\
0
\end{array}\right) \\
& =-\left(\begin{array}{c}
0 \\
0 \\
0 \\
\chi(\sigma) x(\tau)
\end{array}\right)=\iota_{*}(-(\chi \cup x)(\sigma, \tau)) .
\end{aligned}
$$

Thus the coboundary is $-(\chi \cup x)$, which is equal to $x \cup \chi$ by skew-symmetry of of the cup product on $H^{1}$. Theorem 2.1 implies that $\chi$ is a non-zero multiple of the Kummer character associated with $\eta_{p-3}$. Furthermore, $x$ is the Kummer character associated with $\eta_{p-r+3}$. Hence, under condition (3), the coboundary is nontrivial.

Finally, non-triviality of the coboundary implies non-triviality in $\amalg$ by virtue of the commutative diagram with exact rows and columns:

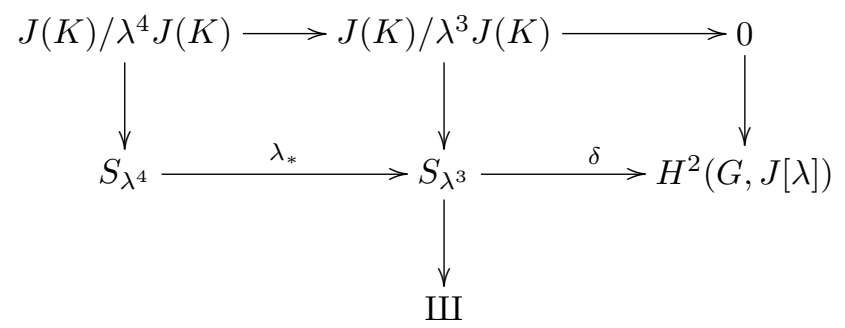

This concludes the proof of the theorem.

\section{Concluding remarks}

From consideration of the action of $\operatorname{Gal}\left(\mathbb{Q}\left(\mu_{p}\right) / \mathbb{Q}\right)$, one does not expect often any overlap between the non-trivial elements of $\amalg$ produced by Theorems 1.1, 1.2 and 1.3. Furthermore, in many cases we can increase a lower bound on the order 
of $\amalg$ using the following result, which depends on showing the triviality in many cases of the Cassels pairing between $\amalg\left[\lambda^{2}\right]$ and $\amalg[\lambda]$.

TheOREM 5.1. [MT03, Theorem 1.2] Suppose that $F_{s}$ is wild non-split or tame and either $p \equiv 1(\bmod 4)$ and $p \nmid B_{(p-1) / 2}$, or $p \equiv 3(\bmod 4)$ and $p \nmid B_{(p-3) / 2}$. Then $\amalg\left[\lambda^{2}\right] / \lambda \amalg\left[\lambda^{3}\right]=0$, that is, $\amalg\left[\lambda^{3}\right]$ is a free module over $\mathbb{Z}[\zeta] /\left(\lambda^{3}\right)$.

Thus, for example, in the case of the curve $y^{691}=x(1-x)$ mentioned in Section 1 , where we found that $\amalg$ contains a subgroup isomorphic to $\mathbb{Z} / 691 \mathbb{Z}$, Theorem 5.1 implies, combined with eigenspace considerations, implies that it contains a subgroup isomorphic to $(\mathbb{Z} / 691 \mathbb{Z})^{3}$. A survey of small irregular primes produces many more examples with large subgroups of $\amalg$. For the curves studied here it seems to be much easier to find elements in $\amalg$ than elements of the Mordell-Weil group.

\section{References}

[Fad61] D. K. Faddeev, Invariants of divisor classes for the curves $x^{k}(1-x)=y^{l}$ in an $l$-adic cyclotomic field, Trudy Mat. Inst. Steklov. 64 (1961), 284-293.

[GR78] Benedict H. Gross and David E. Rohrlich, Some results on the Mordell-Weil group of the jacobian of the Fermat curve, Invent. Math. 44 (1978), 201-224.

[Gre81] Ralph Greenberg, On the Jacobian variety of some algebraic curves, Compositio Math. 42 (1980/81), no. 3, 345-359.

[McC88] William G. McCallum, On the Shafarevich-Tate group of the Jacobian of a quotient of the Fermat curve, Invent. Math. 93 (1988), no. 3, 637-666.

[McC92] William McCallum, The arithmetic of Fermat curves, Math. Ann. 294 (1992), 503-511.

[MS03] William G. McCallum and Romyar T. Sharifi, A cup product in the Galois cohomology of number fields, Duke Math. J. 120 (2003), no. 2, 269-310. MR MR2019977 (2004j:11136)

[MT03] William G. McCallum and Pavlos Tzermias, On Shafarevich-Tate groups and the arithmetic of Fermat curves, Number theory and algebraic geometry, London Math. Soc. Lecture Note Ser., vol. 303, Cambridge Univ. Press, Cambridge, 2003, pp. 203-226.

[Sha07] Romyar T. Sharifi, Iwasawa theory and the Eisenstein ideal, Duke Math. J. 137 (2007), no. 1, 63-101.

[Was97] Lawrence Washington, Introduction to cyclotomic fields, 2nd ed., Springer-Verlag, New York, 1997.

Department of Mathematics, California State University, Chico, CA 95929, USA

E-mail address: benjamin.levitt@gmail.com

Department of Mathematics, University of Arizona, Tucson, AZ 85718, USA

E-mail address: wmc@math.arizona.edu

URL: http://math.arizona.edu/ wmc 